\title{
Chaotic Phenomena in Three Settings: Large, Noisy, and Out of Equilibrium
}

\author{
Lai-Sang Young ${ }^{1}$ \\ Courant Institute of Mathematical Sciences \\ New York University
}

\begin{abstract}
Three directions of research are proposed. The first two concern the creation, detection and calibration of chaos in (1) high or infinite dimensional systems and (2) stochastically perturbed systems. It is suggested that existing ideas from parts of (finite dimensional) hyperbolic theory be extended to these settings. The third topic has a statistical mechanics flavor. A better understanding of nonequilibrium steady states of chaotic Hamiltonian models is sought.
\end{abstract}

In this paper, I will discuss three directions - general directions rather than specific problems - that I believe are relevant and important. I have hinted at these directions in the title; let me elaborate a little more here. Our focus throughout will be on complex dynamical phenomena, including mechanisms for producing chaos, statistics of relevant observations, and significant changes in dynamical landscapes. We seek both a theoretical understanding and ways to connect mathematical ideas to concrete models. By "large" systems, I have in mind high and possibly infinite-dimensional systems; "noisy" systems are processes governed by rules with stochastic as well as deterministic components; and by systems that are "out of equilibrium", I have in mind systems that are driven out of their natural states, possibly by being put in contact with larger sources (or "heat baths").

These directions are far from novel; related articles have occupied many pages of Nonlinearity. But with hyperbolic theory reaching maturity, there is all the more reason for renewed interest. Hyperbolic dynamics is the part of dynamical systems that studies chaotic behavior primarily in finite dimensions. The subject took off with a very successful axiomatic approach (Smale's Axiom A) in the 1960s, and has undergone a great deal of expansion and generalization some of which I will discuss. Over the years, quite sophisticated techniques have been developed and beautiful results obtained, yet the research topics of large, random, and out-of-equilibrium systems have remained wide open. To be sure, chaotic phenomena in these settings have a different flavor and will require different approaches. I challenge here experts in hyperbolic theory and other dynamicists to apply their knowledge - and to acquire whatever additional skills that may be needed - to explore this less charted terrain.

In the pages to follow, I will discuss in more detail the types of systems and questions I have in mind. Since these are vast topics, I can only illustrate through

\footnotetext{
${ }^{1}$ This research is partially supported by a grant from the NSF
} 
examples, and it is inevitable that I will draw from my own experience. Yet through these examples, I hope the challenge will be made clear.

\section{Chaotic Behavior in Large Dynamical Systems}

To illustrate what I mean by "large" dynamical systems, I will describe separately two classes of examples before proceeding to a discussion of how existing hyperbolic theory may serve as a guide for future work.

\section{A. Infinite dimensional systems defined by evolutionary PDEs}

Setting. Consider, for definiteness, a dissipative evolutionary partial differential equation with specified boundary conditions. Under suitable conditions, such an equation leads to a well defined dynamical system defined by

$$
\frac{d u(t)}{d t}=F(u(t)), \quad u(t) \in \mathbb{H},
$$

where $\mathbb{H}$ is a Hilbert space of functions on $\Omega \subset \mathbb{R}^{d}$, the domain in which the physical phenomenon occurs, and $u(t)$ describes the state of the system at time $t$. To use dynamical systems methods, one needs to track simultaneously the evolution of large sets of solutions (as opposed to focusing on one solution at a time), so the semi-group point of view is useful, i.e., we consider $S(t), t>0$, where $t \mapsto u(t)=S(t)\left(u_{0}\right)$ is the solution with $u(0)=u_{0}$. It is usually necessary to restrict to smaller subspaces $X \subset \mathbb{H}$ to obtain the needed regularity properties for $S(t)$.

Some known results. ${ }^{2}$ For many dissipative systems in physics and mechanics, the existence of absorbing sets and compact global attractors have been shown. These results imply that the dynamics of interest are captured on relatively small sets; see e.g. $[\mathrm{BV}, \mathrm{H}, \mathrm{T}]$. Basic tools of analysis such as center and stable manifolds have also been developed for infinite dimensional systems.

These and many other works are concerned primarily with upper bounds on the complexity of the system, which are important given the infinite dimensionality, but they do not provide a great deal of information on the structures of the attracting sets or the type of dynamics, such as whether or not they are chaotic. ${ }^{3}$ A number of other results in the literature prove the presence of complicated behavior in various PDEs as manifested in the existence of horseshoes and homoclinic solutions (which suggest chaos); see e.g. [HM, LMSW]. In a recent work, my co-authors and I proved a stronger form of chaos characterized by the existence of "strange attractors" with

\footnotetext{
${ }^{2}$ Here as elsewhere in the paper, I need to mention some known facts to lead up to the discussion of proposed directions. I want to stress that the references given are not intended to be a complete or unbiased account of what is known.

3 "Chaos" in this paper refers exclusively to chaos in the time evolution of the system; it is not to be confused with spatial chaos.
} 
positive Lyapunov exponents "almost everywhere" in open sets of initial conditions. The setting is that of periodic kicking of a system shortly after it undergoes a Hopf bifurcation [LWY]. This result is not specific to any PDE; it is about a dynamical phenomenon that can occur in any dimension, including infinite dimensions.

\section{B. Composite systems via coupling of many small constituent systems}

I have in mind here a loosely defined class of dynamical systems which I will not try to make precise but will instead illustrate via three examples.

Coupled maps systems. These systems are discrete-time analogs of reaction-diffusion processes. A typical setup is

$$
x_{i}(n+1)=(1-\varepsilon) f\left(x_{i}(n)\right)+\frac{\varepsilon}{2}\left(f\left(x_{i-1}(n)\right)+f\left(x_{i+1}(n)\right)\right)
$$

where $i \in \mathbb{Z}$ denotes the site, $n=0,1,2, \cdots$ is discrete time, $x_{i}(n)$ takes values in $\mathbb{R}^{d}, f: \mathbb{R}^{d} \rightarrow \mathbb{R}^{d}$ defines the local dynamics, and $\varepsilon$ is the coupling constant. For a summary of the state of the art as of 2005, see [CF]. When $f$ has well controlled chaotic properties, (e.g., it is expanding, piecewise expanding or hyperbolic), and $\varepsilon$ is small, it has been well documented that these properties are inherited by the coupled dynamical system. Other situations have been studied, but to my knowledge there is not yet a systematic understanding. Some obvious generalizations are: replacing the lattice $\mathbb{Z}$ by more general graphs or networks, finite or infinite, and variable coupling strengths, including those in intermediate ranges (see e.g. $[\mathrm{K}]$ ). These setups can lead to a great variety of behaviors. Local maps that support more than one type of behavior provide candidates for phase transitions in the coupled systems.

Particle dynamics on lattices. I have in mind here extended phase spaces such as those with lattice structures and moving particles whose dynamics are defined by microscopic rules. These models appear naturally in statistical mechanics (see Sect. 3). They are dynamical versions of particle systems in probability, which have a similar setup but often without the internal degrees of freedom at each site. Examples of results in this direction are [CD, KY, Le].

Networks of coupled oscillators. These systems appear naturally as paradigms or simplified models in the physical and biological sciences. In Neuroscience, for example, intrinsically active neurons are sometimes modeled as oscillators. As a neuron "spikes", it sends a signal to other neurons "downstream", possibly modifying the phase or behavior of the receiving neurons (see e.g. [GK]). These networks are usually finite in size, but can be very large. Not surprisingly, much attention has been paid to simpler types of behaviors such as synchronization, while more complex dynamical behaviors are known to occur but not as well recognized. Viewed as models of specific situations, the interpretation (and relevance) of chaotic phenomena will depend on scientific context, but these systems are so ubiquitous that I believe they are excellent training models. 


\section{Relevant facts from hyperbolic theory}

In low dimensional systems, it is generally accepted that chaos is created via stretch-and-fold mechanisms. Does this continue to hold in higher dimensions? I will discuss below different types of chaos, and give a brief summary of those parts of hyperbolic theory that may be relevant for tackling problems in high dimensions.

1. Invariant cones. The simplest way to guarantee hyperbolicity is a priori knowledge of invariant cones. This method of proof has been used very successfully in geodesic flows on manifolds of negative curvature, billiards, hard balls etc. In the examples above, invariant cones are defined everywhere. When they are available on only part of the phase space, they can often still be used to prove hyperbolicity on some invariant sets, such as horseshoes. Global invariant cones are used in proving the existence of inertial manifolds in infinite dimensions.

2. Horseshoes. The existence of complex behavior is often symbolized by the presence of horseshoes. Having horseshoes is a geometric condition that can be proved by identifying directly the set that folds on itself. At other times it is proved via the existence of transversal homoclinic points, which, in finite dimensions is well known to imply the presence of horseshoes. In infinite dimensions, more care is needed due to complications caused by the noninvertbility of the map.

While they symbolize the existence of complex behavior, it should be pointed out that the presence of horseshoes in itself does not imply that "most" orbits are chaotic, or that any observed chaos will persist. It is entirely possible for horseshoes and sinks (or stable equilibria) to coexist. When that happens, we say the system has transient chaos: Starting from an initial condition near a horseshoe, the trajectory is likely to appear chaotic for some time as it follows the chaotic dynamics on the horseshoe. But since only a very small set of points are actually attracted to the horseshoe, a randomly chosen orbit is likely to leave the vicinity of the horseshoe after some time. In the case of transient chaos, it is eventually attracted to a sink.

3. Strange attractors and SRB measures. In finite dimensions, positive Lebesgue measure sets are often equated with observable events. With this interpretation, a form of chaos that is both observable and sustained in time (i.e., not transient) is guaranteed by having positive Lyapunov exponents for a positive Lebesgue measure set of initial conditions. This can happen for volume-preserving systems as well as dissipative ones. In the latter, there is an attractor, and the orbits in question lie in its basin of attraction. As a shorthand, I will refer to such a dynamical picture as a strange attractor. The only known way to establish the presence of strange attractors is to show that the system admits a special invariant measure called an SRB measure (see $[\mathrm{Y}]$ for more information). All this, however, relies heavily on the role of Lebesgue measure, leaving the situation in infinite dimensions unclear. In a recent work [LWY], my co-authors and I explained why SRB measures are likely to retain their important role for large classes of dissipative parabolic PDEs. 
4. Center manifolds. I wonder if they can be exploited more effectively in situations that are finite dimensional in character - such as in systems defined by parabolic PDEs - even where no global center manifolds exist.

5. High $v s$ low dimensional chaos. I refer here to the number of degrees of freedom involved in the creation of chaos, or the number of positive Lyapunov exponents. First, observe that if one equates chaos with unpredictability, i.e., entropy, then in infinite dimensional systems, hyperbolicity is not a prerequisite for chaos. With regard to detecting hyperbolicity, except where invariant cones are present, little is known about high dimensional chaos. At the other end of the spectrum, there has been some success in rank one chaos, i.e., the study of strange attractors with a single direction of instability [WY]. These attractors are as simple as strange attractors can be; they occur near the "boundary" of chaos, soon after stability is lost. At the present time, we are more equipped to deal with low dimensional chaos, which occurs naturally as we have discussed.

\section{Chaos in Random Dynamical Systems}

A second direction in which I would like to see more progress is chaotic phenomena in random dynamical systems, by which I include general stochastically driven systems as well as deterministic systems perturbed by small amounts of random noise modeling uncontrolled fluctuations. My reasons for focusing on these systems are that (1) they occur naturally, and (2) random systems are better behaved than purely deterministic systems, as we will explain.

The formal mathematical framework I have in mind is that of compositions of i.i.d. sequences of smooth maps. More precisely, one fixes a probability measure $\nu$ on $\mathcal{M}(X)$, the space of maps from a manifold $X$ to itself, and consider the dynamics generated by compositions of the form

$$
f^{(n)}=f_{n} \circ \cdots \circ f_{2} \circ f_{1}, \quad n=1,2, \cdots,
$$

where $f_{1}, f_{2}, \cdots$ are chosen independently with respect to $\nu$. For brevity, we will refer to this setup as random maps. We are especially interested in the case where the $f_{i}$ are smooth.

Random maps arise naturally. In the discrete-time case, given a map $f: X \rightarrow X$, one often represents a random perturbation of $f$ as a Markov chain whose transition probabilities are given by $P(A \mid x)=Q_{f(x)}(A)$ where $\left\{Q_{x}, x \in X\right\}$ is a family of probability distributions on $X$ with $Q_{x}$ concentrated near $x$. Not all Markov chains of this type can be represented as random smooth maps, but in many situations, such representations are feasible. Sometimes all the $f_{i}$ can be taken close to $f$ in some $C^{r}$ topology. In the case of continuous time, it is well known that an SDE of the form

$$
d x_{t}=a\left(x_{t}\right) d t+\sum_{i=i}^{n} b_{i}(t) \circ d W_{t}^{i}
$$


gives rise to a stochastic flow of diffeomorphisms. Here $a(\cdot)$ and $b(\cdot)$ are smooth enough vector fields, $W_{t}^{1}, \cdots, W_{t}^{n}$ is a standard Brownian motion, and the Stratonovich integral is assumed.

By chaos in random maps, I refer to pathwise chaos, that is to say, chaotic behavior that arise by following individual sample paths. When the $f_{i}$ are smooth, Lyapunov exponents $\left\{\lambda_{i}\right\}$ for the random system are well defined and are nonrandom, i.e., corresponding to each ergodic stationary measure, the system is described by a finite set of numbers. The following dichotomy tells us that the dynamical picture corresponding to positive and negative Lyapunov exponents are very different: Letting $\omega=\left\{f_{i}\right\}_{i=\infty}^{\infty}$, we may disintegrate any stationary measure $\mu$ into a family of sample measures $\left\{\mu_{\omega}\right\}$ defined by conditioning on the past. That is to say, $\mu_{\omega}=\lim _{n \rightarrow \infty}\left(f_{-1} \circ f_{-2} \circ \cdots \circ f_{-n}\right)_{*} \mu$. Omitting technical conditions, we state the following theorem:

Theorem Assume $\mu$ is ergodic with Lyapunov exponents $\left\{\lambda_{i}\right\}$. Then almost surely:

(1) [LeJ] If $\lambda_{i}<0$ for all $i$, then $\mu_{\omega}$ is supported on a finite set of points.

(2) [LeY] If $\mu$ has a density, and $\lambda_{i}>0$ for some $i$, then the $\mu_{\omega}$ are random SRB measures, i.e., they have smooth conditional densities on unstable manifolds.

These two results together tell us that except when the largest $\lambda_{i}$ is equal to 0 , the system is either highly nonchaotic, with all solutions coalescing into a random sink, or it has a random strange attractor. This clear dichomoty, together with the fact that Lyapunov exponents vary continuously under mild conditions, is to be contrasted with iterations of single maps, where the dynamical picture can vary in a very complicated way with parameters. As an example, consider the logistic family $f_{a}(x)=1-a x^{2}, x \in$ $[-1,1]$. In this much studied example, sinks occur for an open and dense set of parameters, while for a positive measure set $a, f_{a}$ has a positive Lyapunov exponent; see [Ly]. For another example of what I meant by the world of randomly perturbed dynamics being nicer, see [LiY].

Yet in spite of these properties of random maps, few techniques are available for proving the positivity of Lyapunov exponents. In the purely deterministic case, what lies behind the difficulty in proving the positivity of Lyapunov exponents is the fact that norms of matrices are submultiplicative. That is to say, if each of $A_{1}, A_{2}, \cdots, A_{n}$ is hyperbolic, it does not imply that their product is hyperbolic. This is because directions that have been expanded by one linear map may be contracted by another, and vice versa. In other words, even when one is able to prove exponential growth for individual segments of an orbit, there is no guarantee that when concatenated, the orbit will have a positive Lyapunov exponent. This problem is present for random maps as for iterations of single maps, but one would expect that randomness in the directions of tangent vectors will make certain averaging arguments possible.

To develop basic techniques, it is probably necessary to begin with specific examples that are "quite obvious" (but for which no proofs exist at present). An eventual goal is to show that if the geometry of a random map suggests that it may have a 
positive Lyapunov exponent, then it actually does. That is to say, if a system has expansions on a large enough portion of its phase space and the randomness is strong enough to overcome conspiracies to form sinks (through the "wrong" combination of expansion and contraction described in the last paragraph), then positive Lyapunov exponents are guaranteed. This type of results ought to be true.

\section{$3 \quad$ Nonequlibrium Dynamics}

Many of the ideas in this section are borrowed from nonequilibrium statistical mechanics. Attempts to explain heat conduction, for example, has led to interesting and challenging problems in dynamical systems. Because of their wide range of applicability, I propose to study these questions in broader contexts, to view them as generic problems in dynamical systems with motivations from physics.

Consider a system defined by a map, a flow, or a stochastic process on a phase space $X$, with no interaction with "the outside". I call this a closed system, or a system in isolation. Much of dynamical systems theory as it exists today is concerned with the study of closed systems. By contrast, an open system is one that has contact with some other systems external to the one in question. This contact permits exchanges of various kinds. In physics, they are usually in the form of matter (mass), energy or momentum. In a general study, one may think of it as some abstract quantity that may enter or leave the system in question. The external systems can take on various forms. It can, for example, be another system of a similar type. An often-encountered situation is when the system in question is in contact with a much larger system, one that is infinite, or large enough that it is not affected by the exchanges that take place. I will refer to these large (insensitive) systems as heat baths.

Let us assume, for present purposes, that when a system is left alone, it relaxes to a "natural state". We then say a system is out of equilibrium when it is not in one of its natural states. This can be arranged, for example, by putting it in contact with multiple heat baths at different "temperatures". Steady states reached under such circumstances are called nonequilibrium steady states (NESS). The simplest example is a rod the two ends of which are maintained at two different temperatures. As in statistical mechanics, the challenges are to determine the properties of NESS for systems that are out of equilibrium, and to explain macroscopic observations in terms of the microscopic rules that define the system. It is not that transient behavior is uninteresting; on the contrary, the routes to NESS are known to be complex and rich. But in this brief discussion, let us focus only on NESS. We will assume the system is large enough that one may think in terms of infinite-volume limits. This is roughly what I mean by nonequilibrium dynamics.

I think it is useful to have a concrete example to which I can point - even though the ideas I want to discuss are general and not at all specific to this example.

Example. The following is a toy model of particle and energy transport through 
a one-dimensional chain brought on by unequal boundary conditions. The physical domain of this Hamiltonian system is a linear array of $N$ identical "cells". The energy level in each cell is symbolically represented by the kinetic energy of a rotating disk. Particles enter and leave the chain from the two ends. As they move about in the chain bouncing off the walls of the cells, the particles do not interact with each other, but exchange energy with the rotating disks as they collide (by e.g., swapping tangential components of their momenta). The system is determined by (i) the rates at which particles are injected at the two ends, and (ii) the kinetic energy distributions of the injected particles. Systems along these lines were studied in [EY, LLM].

As noted at the beginning of this section, the discussion to follow is based on ideas that are far from my own, although I will give them a bit of a dynamical slant. I will assume throughout that the system in question is out of equilibrium. In the example above, this means boundary conditions are fixed in such a way that (i) and (ii) are unequal at the two ends. For example, the particles that enter from the right may be "hotter", and there is a flow of energy from right to left. For mathematicians, the first questions are those of existence and uniqueness of NESS.

Next we identify the macroscopic quantities of interest. In the example above, this may be particle density or the profile of mean energies (as stored in the rotating disks). To get a handle on these quantities, it is necessary to understand the local picture, for it is the microdynamics that determine the invariant measure. Consider then a small piece of physical space located in the interior of the domain. Even when boundary conditions are unequal, gradients should be nearly zero inside a tiny area, so it may not be unreasonable to think of the system restricted to this tiny area as being "in equilibrium". But what is the nature of this equilibrium? In our example, this amounts to picking a finite number of contiguous cells, located, say, a third of the way from the left end of the chain, and asking about the marginal of the invariant measure. Clearly, what goes on inside this subsystem is determined by what is injected into it, so the answer is determined by the energy and particle fluxes through the one-third point of the (long) chain given its boundary conditions.... There is a host of related questions, such as correlations, large deviations, and so on.

Different types of dynamical interactions are likely to lead to different answers. In our example, if we neglect (for a moment) the interaction between particles and disks, then the problem is reduced to that of a billiard flow in a single cell, and we know from billiard theory that the shapes of the cells play a crucial role: for example, if the walls of the cells are concave (or scattering), then the movement of a particle along the chain, when properly scaled, will tend to Brownian motion [BSC], while certain polygonal walls can lead to anomalous diffusion. Now the energy exchange between particles and disks cannot be neglected; that would be simplifying away the most crucial ingredient of the system. It is easy to see that properties of the invariant measure will depend strongly on the nature of this interaction. The more "chaotic" it is, the more the evolution of the energy will mimick a random process, and there has been some success in the treatment of stochastic models of this type (see e.g. [KMP, 
$\mathrm{KL}])$. Closing the gap between deterministic and stochastic dynamics is too much to hope for. Still, I believe some headway can be made for dynamical models, although one should perhaps aim for rougher and more partial qualitative results.

To summarize, in the first two sections, I was looking for mechanisms for producing chaos and ways to detect it. Here I am trying to leverage what we know about chaotic systems to shed light on nonequilibrium dynamics.

\section{Remarks on Methodology}

I strongly advocate the combined use of analysis and simulations to gain insight into the types of dynamical systems discussed in this article, especially those discussed in Sects. 1 and 3. When the degree of complexity of a system is sufficiently high, it is natural to turn to numerics for exploratory purposes. But what I have in mind for the role of numerical computation goes beyond that. There will be many exceptions to the view I am about to express, but it seems to me that for these vastly complex systems, while analytic methods alone can give results that are general and theoretical - and such results are absolutely vital - it is hard to obtain a concrete, more "quantitative" kind of understanding without the aid of simulations. I am, therefore, in favor of multiple routes of attack using a range of methods from pure analysis to simulations, with the gains from one used to the benefit of the others.

\section{References}

[BV] A.V. Babin and M.I. Vishik. Attractors of Evolution Equations, North-Holland, Amsterdam, (1992).

[BSC] L. Bunimovich, Ya.G. Sinai, and N. Chernov, Statistical properties of twodimensional hyperbolic billiards, Russ. Math. Surv. 46 (1991), 47106.

[CF] Dynamics of Coupled Map Lattices and or related specially extended systems, Ed. J.-R. Chazottes and B. Fernandez, Lecture Notes in Physics 671, Springer-Verlag (2005).

[CD] N. Chernov and D. Dolgopyat, Galton board: limit theorems and recurrence, 2007 preprint.

[EY] J.-P. Eckmann and L.-S. Young, Nonequilibrium energy profiles for a class of 1-D models, Comm. Math. Phys. 262 (2006), 237-267.

[GK] W. Gerstner and W. Kistler, Spiking Neuron Models, Cambridge Univ. Press (2002)

[H] J. Hale, Asymptotic behavior of dissipative systems. Mathematical Surveys and Monographs, 25. American Mathematical Society, Providence, RI (1988).

[HM] P. Holmes and J. Marsden. A partial differential equation with infinitely many periodic orbits: chaotic oscillations of a forced beam, Arch. Rat. Mech. Anal., 76 (1981), 135-166. 
[KY] E. Kobre and L.-S. Young, Extended systems with deterministic local dynamics and random jumps, Commun. Math. Phys. 275, no.3 (2007), 709-720.

[K] J. Koiller, Ph.D. thesis, Courant Institute of Math. Sc., New York Univ., (2008).

[KL] C. Kipnis, C. Landim, Scaling Limits of Interacting Particle Systems, Berlin: Springer-Verlag (1999)

[KMP] C. Kipnis, C. Marchioro, and E. Presutti, Heat flow in an exactly solvable model, J. Stat. Phys., 27 (1982), 65-74

[LLM] H. Larralde, F. Leyvraz, and C. Mejía-Monasterio, Transport properties of a modified Lorentz gas, J. Stat. Phys. 113 (2003), 197-231.

[LeJ] Y. Le Jan, Équilibre statistique pour les produits de diffeomorphismes aléatoires indépendants, Ann. Inst. H. Poincaré Probab. Statist. 23 (1987), no.1, 111-120.

[LeY] F. Ledrappier and L.-S. Young, Entropy formula for random transformations, Prob. Th. Rel. Fields, 80, (1988), 217-240.

[Le] M. Lenci. Typicality of recurrence for Lorentz gases, Erg. Th. \& Dynam. Sys. 26 (2006), no. $3,799-820$.

[LMSW] Y. Li, D. W. McLaughlin, J. Shatah, and S. Wiggins. Persistent homoclinic orbits for a perturbed nonlinear Schrödinger equation, Comm Pure Appl Math, 49(11) (1996), $1175-1255$.

[LiY] K. Lin and L.-S. Young, Shear-induced chaos, Nonlinearity, 21, (2008) 899-922.

[LWY] K. Lu, Q. Wang, and L.-S. Young. Strange attractors for periodically forced parabolic equations, 2007 preprint.

[Ly] M. Lyubich, Almost every real quadratic map is either regular or stochastic, Ann. Math., Vol. 156 (2002), 1-78.

[T] R. Temam. Infinite Dimensional Dynamical Systems in Mechanics and Physics, Applied Math. Sc., Springer-Verlag, 68 (1997).

[WY] Q. Wang and L.-S. Young, Toward a theory of rank one attractors, Ann. Math., Vol. 167, No.2 (2008), 349-480.

[Y] L.-S. Young, What are SRB measures, and which dynamical systems have them? J. Stat. Phys. 108 (2002), 733-754. 